\title{
Clinical Outcomes of Conservative Treatment and Arthroscopic Repair of Rotator Cuff Tears: A Retrospective Observational Study
}

\author{
Woo Hyung Lee, $\mathrm{MD}^{1,2}$, Hyun Kyung $\mathrm{Do}, \mathrm{MD}^{2}$, Joong Hoon Lee, MD², Bo Ram Kim², \\ Jee Hyun $\mathrm{Noh}^{2}$, Soo Hyun $\mathrm{Choi}^{2}$, Sun Gun Chung, MD, $\mathrm{PhD}^{3}$, Shi-Uk Lee, MD, $\mathrm{PhD}^{4}$, \\ Ji Eun Choi, PhD $^{1}$, Seihee Kim ${ }^{1}$, Min Jee Kim ${ }^{1}$, Jae-Young Lim, MD, PhD ${ }^{1,2}$ \\ ${ }^{1}$ National Evidence-Based Healthcare Collaborating Agency, Seoul; \\ ${ }^{2}$ Department of Rehabilitation Medicine, Seoul National University Bundang Hospital, Seongnam; \\ ${ }^{3}$ Department of Rehabilitation Medicine, Seoul National University Hospital, Seoul; \\ ${ }^{4}$ Department of Rehabilitation Medicine, Seoul National University Boramae Medical Center, Seoul, Korea
}

Objective To compare the clinical outcomes following conservative treatment and arthroscopic repair in patients with a rotator cuff tear.

Methods In this retrospective study, patients aged $>50$ years with a symptomatic rotator cuff tear were reviewed. The rotator cuff tendons were evaluated using ultrasonography, shoulder magnetic resonance imaging or MR arthrography, and the patients with either a high-grade partial-thickness or small-to-medium-sized $(\leq 3 \mathrm{~cm})$ fullthickness tear were included in this study. The primary outcome measures were a pain assessment score and range of motion (ROM) at 1-year follow-up. The secondary outcomes were the rate of tear progression or retear along with the rate of symptom aggravation after the treatments.

Results A total of 357 patients were enrolled, including 183 patients that received conservative treatment and 174 patients who received an arthroscopic repair. The pain assessment score $(\mathrm{p}<0.001)$ and the ROM in forward flexion $(p<0.001)$ were significantly improved in both groups. The ROM in internal rotation did not significantly change after conservative treatment and arthroscopic repair. The pain assessment score and ROM were not significantly different between the two groups. Retear was observed in $9.6 \%$ of patients who had an arthroscopic repair and tear progression was found in $6.7 \%$ of those who underwent conservative treatment. The proportion of aggravation for pain and ROM did not significantly differ between the two groups.

Conclusion The effectiveness of conservative treatment is not inferior to arthroscopic repair for patients $>50$ years old with a less than medium-sized rotator cuff tear in a 1-year follow-up period. Further study is warranted to find the optimal combination of conservative treatment for a symptomatic rotator cuff tear.

Keywords Arthroscopy, Conservative treatment, Rotator cuff, Tendon injuries, Treatment outcome

Received June 1, 2015; Accepted August 14, 2015

Corresponding author: Jae-Young Lim

Department of Rehabilitation Medicine, Seoul National University Bundang Hospital, 82 Gumi-ro 173beon-gil, Bundang-gu, Seongnam 13620, Korea Tel: +82-31-787-7732, Fax: +82-31-787-4051, E-mail: drlim1@snu.ac.kr

(c) This is an open-access article distributed under the terms of the Creative Commons Attribution Non-Commercial License (http://creativecommons.org/ licenses/by-nc/4.0) which permits unrestricted noncommercial use, distribution, and reproduction in any medium, provided the original work is properly cited. Copyright ( 2016 by Korean Academy of Rehabilitation Medicine 


\section{INTRODUCTION}

A rotator cuff disorder is a major cause of shoulder pain and disability, accounting for $20 \%$ to $50 \%$ of cases in older adults [1]. This proportion may increase as the population ages [2]. Even though the medical needs of the symptomatic rotator cuff tear have increased rapidly, it is difficult to determine the appropriate treatment methods that should be used in clinical practice. Many different types of treatment methods can be applied to this disorder including pain medication, activity modification, manual therapy, supervised exercise, corticosteroid injection and surgical repair [3]. Natural history and comparative outcome of conservative and surgical treatments are not coincident among several related studies. It has been argued in some studies that conservative treatment does not promote tendon healing and that the size of rotator cuff tear may progress [4-7]. According to these studies, surgical repair is beneficial in improving tendon healing. However, other studies have shown that more than $90 \%$ of patients experienced symptomatic and functional recovery from a symptomatic rotator cuff tear without surgical repair [8]. Furthermore, the severity of the rotator cuff tear and symptoms of the patients are not known to be correlated [9]. As these controversial results have shown, there are still numerous arguments for what methods are optimal for management of a symptomatic rotator cuff tear.

Several studies have reported that surgical repair showed good clinical outcomes when surgical repair techniques developed from invasive open surgery to the less invasive mini-open or arthroscopic surgeries [10-12]. On the other hand, recent prospective randomized studies comparing surgical and conservative treatments reported that the difference of outcomes were not clinically significant between each treatment $[13,14]$, which implies that this issue is an ongoing controversy. In Korea, numerous studies on shoulder disorders have appeared in recent years that show an increased interest and a high level of treatment, but a study on the comparison or appropriateness of the treatment has not yet been performed. There has been a paucity of studies that reflect real clinical practice in which conservative treatment is comprised of heterogeneous combinations of treatment methods, and where most patients are middleor advanced-aged. Therefore, the aim of this study was to compare the clinical outcomes following conservative treatment and arthroscopic repair of a rotator cuff tear in patients $>50$ years old at middle and advanced ages.

\section{MATERIALS AND METHODS}

\section{Patient data sources and collection}

Three medical centers were queried regarding patients

Search terms:

Patient data source

1) Diagnosis: 'rotator cuff tear'

2) Assessment methods: 'shoulder ultrasonography' or 'shoulder MRI' or 'shoulder MR arthrography' : total 17,286 patients

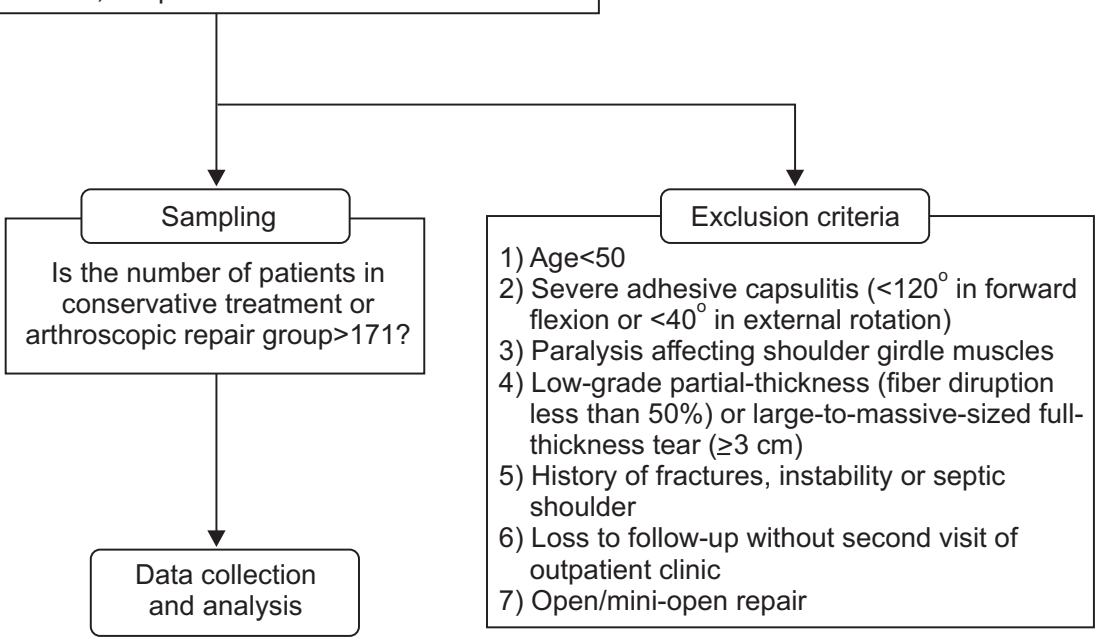

Fig. 1. The flow chart of the patient data source, sampling and analysis. 
with a rotator cuff tear from January 2008 to January 2013. Fig. 1 shows the flow chart of data source, sampling and analysis. A total of 17,286 patients were identified using search terms. Search terms were comprised of diagnosis as 'rotator cuff tear' and assessment methods as 'shoulder ultrasonography', 'shoulder magnetic resonance imaging (MRI)' or 'shoulder MR arthrography'. Among the patients who were identified using these search terms, the patients who had a high-grade partial-thickness (fiber disruption more than $50 \%$ ) or small-to-medium-sized full-thickness tear $(\leq 3 \mathrm{~cm})$ were extracted randomly in this study. Patients were excluded if they met any of the following criteria: 1) less than 50 years of age, 2) severe adhesive capsulitis $\left(<120^{\circ}\right.$ in forward flexion or $<40^{\circ}$ in external rotation), 3) paralysis affecting shoulder girdle muscles, 4) low-grade partial-thickness (fiber disruption less than 50\%) or large-to-massive-sized full-thickness tear $(\geq 3 \mathrm{~cm})$ preexisted, 5 ) history of fractures, instability or septic shoulder, 6) open or mini-open repair or 7) loss to follow-up without second visit of outpatient clinic. After the patient list was determined, convenient sampling was conducted for each conservative and arthroscopic repair group. Demographic data for the patients in the final list were then collected in the medical records regarding sex, age, tear on dominant side, pain onset and existence of trauma history. Age and pain onset were determined based on the time at the initial visit of the outpatient clinic. Acute onset of pain was defined as occurring less than three months before the initial visit. This study was approved at three medical centers and an agency to which all the authors belonged.

Evaluation of rotator cuff muscles and clinical outcomes

Rotator cuff tendons were evaluated using shoulder ultrasonography, shoulder MRI or MR arthrography in terms of tear thickness, size and fatty degeneration. A partial thickness tear was classified as 'low grade' if less than $50 \%$ fiber was disrupted and 'high grade', if more than $50 \%$ was disrupted. A full thickness tear was classified as small $(<1 \mathrm{~cm})$, medium $(1-3 \mathrm{~cm})$, large $(3-5 \mathrm{~cm})$ and massive ( $>5 \mathrm{~cm}$ or more than two tendons involved) [15]. The tear size of rotator cuff tendons was determined by measuring the length of retraction and width in each sagittal and coronal plane seen on ultrasonography, MRI or MR arthrography images (Fig. 2). Fatty degeneration was assessed using shoulder MRI or MR arthrography ac- cording to Goutallier classification [16].

Primary outcome measures were a pain assessment score and range of motion (ROM). Pain was evaluated with a numeric rating scale or visual analog scale from 0 to 10. Range of motion was measured passively for forward flexion and internal rotation by an examiner. Internal rotation was measured using a vertebral level at the back. Secondary outcome measures were the rate of tear progression or retear, and the rate of symptom aggravation, after treatment. Aggravation cases were defined as patients with a pain assessment score greater than that of baseline or forward flexion and internal rotation ROM less than that of baseline. The clinical outcomes were measured at initial, 2 to 6-month and 1-year visits of the outpatient clinics and compared between conservative treatment and arthroscopic repair groups. A trained interviewer conducted a telephone interview to assess pain and ROM in patients who were lost to follow-up in a year. During the telephone interview, ROM was measured using several landmarks of the body. In the case of forward flexion, arm elevation next to the ear at the end of arc without neck motion was regarded as full ROM. The buttock, lumbosacral junction, pelvic bone, waist and scapula were used as landmarks to measure internal rotation.

\section{Treatment modalities}

The conservative treatment group included all patients with a rotator cuff tear who were not treated by surgical repair. The treatment comprised of active surveillance, a therapeutic modality, oral pain medication, steroid injection and shoulder exercises. Active surveillance included patient education related to activity modification, control of symptoms or prognosis of the disease, without any medication or intervention [3]. The therapeutic modality included transcutaneous electrical nerve stimulation, heating pad or therapeutic ultrasound. Oral pain medication included non-steroidal anti-inflammatory drugs or opioid analgesic agent such as tramadol/acetaminophen. Intra-articular or subacromial-subdeltoid bursa steroid injection was administered blind or guided using ultrasonography by physiatrists or orthopedic surgeons. The shoulder exercise protocol consisted of active and passive ranges of motion as well as strengthening exercises of the rotator cuff and scapular stabilizing muscles. Surgical treatment was restricted to arthroscopic repair, either with or without acromioplasty, and debridement. 

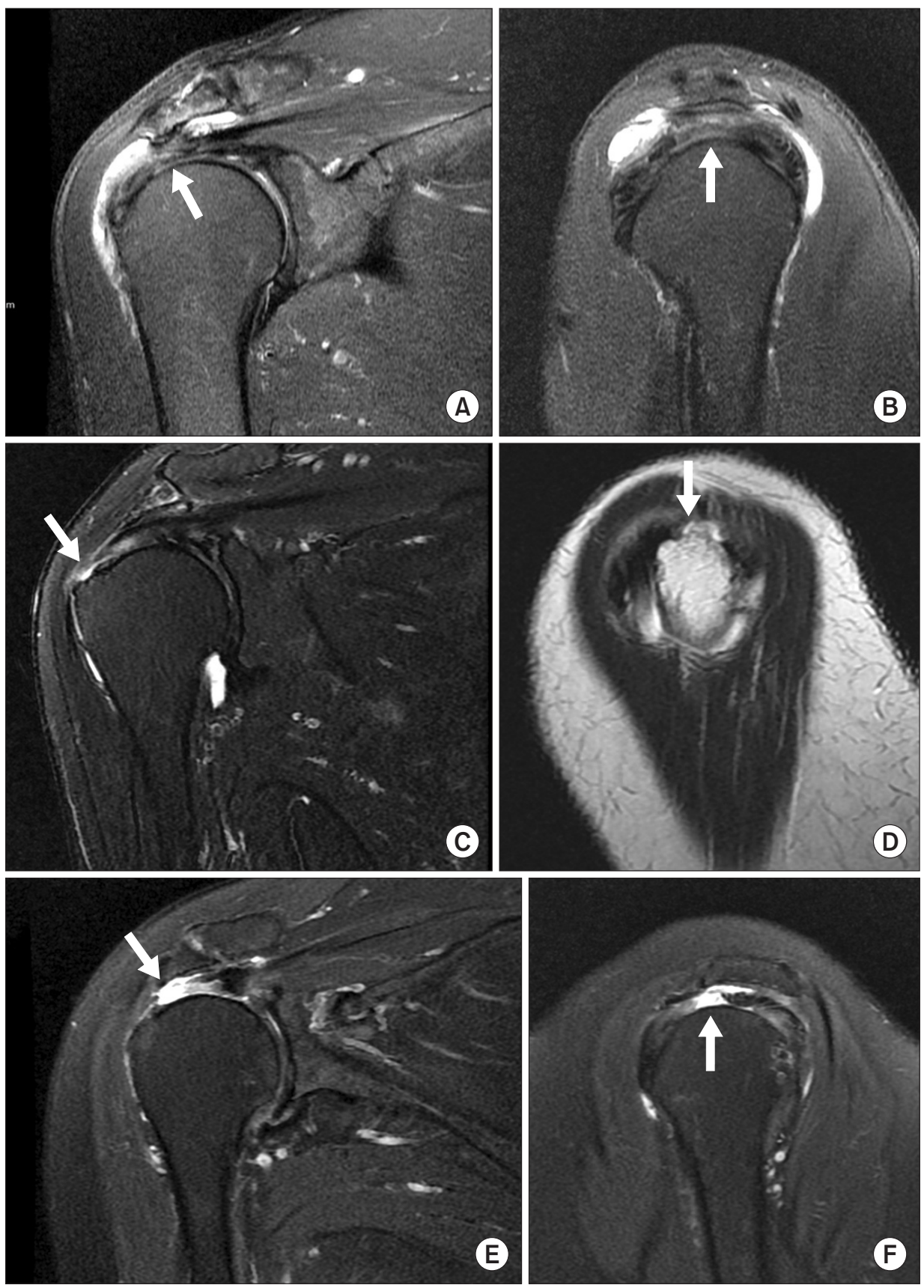

Fig. 2. Typical cases of right supraspinatus tendon tears (arrow) in coronal and sagittal planes of fatsaturated T2-weighted MR images: high-grade partial-thickness (A and B), small- (C and D) and medium-sized full-thickness tears (E and F).
Open and mini-open repair were excluded. After surgical treatment, the arthroscopic repair group was provided with postoperative management that included shoulder exercises, pain medication or corticosteroid injection. The shoulder exercise was initiated to prevent stiffness after 5-6 weeks of postoperative immobilization. Pain medication and corticosteroid injection within 3 months after surgical treatment was regarded as postoperative management in this study.

\section{Statistical analysis}

To reach statistical significance with alpha $=0.05$ and power $=0.9$ for the pain assessment score on the assumed statistical behavior of other previous studies, the number of patients was calculated and 142 patients were needed in the conservative treatment and arthroscopic repair, respectively [14]. By adding $20 \%$ of patients in consideration of the missing data, more than 171 patients were needed in each group. 
Demographic factors including sex, age, tear on dominant side, pain onset, and existence of trauma history were analyzed with a chi-square test. Likewise, tear classification and fatty degeneration at the initial visit were analyzed with the same method. To quantify the extent of internal rotation, each vertebral level was scaled to be a numerical value from 1 to 16 . The sacral level was represented as 1, L5-L1 level as 2-6 and T12-T3 as 7-16. To investigate the simultaneous effects of time and treatment on clinical outcomes, a comparison of clinical outcomes was performed using analysis of variance with repeated measurements and a general linear model. In this model, covariates were used including age, sex, tear on dominant side, trauma history, symptom onset and tear classification. The rate of tear progression in the conservative treatment group or retear in the arthroscopic repair group was analyzed with the chi-square test. The aggravation of clinical outcomes after both treatments was analyzed using the same method. All statistical analyses were performed using SPSS software ver. 19 (SPSS Inc., Chicago, IL, USA). The significance level was set at $\mathrm{p}<0.05$.

\section{RESULTS}

\section{Baseline patient demographics}

Of the patients available for review, 183 who received conservative treatment and 174 who underwent arthroscopic repair met the study inclusion criteria and were enrolled in this study. A telephone interview was conducted for $51.6 \%$ of patients in the conservative treatment group and $50.3 \%$ in the arthroscopic repair group, who were lost to follow-up at 1 -year $(\mathrm{p}=0.85)$. Demographic data of the patients at the baseline shows that there was no significant difference between the two groups for each category (Table 1). The mean age was $64.6 \pm 8.7$ years in the conservative treatment group and $62.3 \pm 6.6$ years in the arthroscopic repair group.

\section{Clinical outcomes}

The changes of pain assessment score and ROM in the 1-year follow-up period are shown in Fig. 3 and Table 2. The pain assessment score and ROM in degrees of forward flexion improved significantly after treatment in both groups $(\mathrm{p}<0.001)$ at follow-up after 1 year, but betweengroup differences at the baseline and at 1 year were not significant. ROM in internal rotation did not signifi- cantly change after both treatments over the year period $(p=0.34)$. The subgroup analysis for high-grade partialthickness and small-to-medium-sized full-thickness tears showed that the pain assessment score as well as forward flexion and internal rotation ROMs were not significantly different after arthroscopic repair or conservative treatment (Tables 3, 4). In the subgroup analysis for the arthroscopic repair group, patients who were prescribed with postoperative pain medication, corticosteroid injection or shoulder exercises did not show any difference according to their pain assessment score and ROM for forward flexion and internal rotation as compared with non-prescribed patients.

Rotator cuff tendons were reevaluated for 125 patients $(71.8 \%)$ in the arthroscopic repair group and 45 patients $(24.6 \%)$ in conservative treatment group at follow-up 1 year later. There were 12 cases $(9.6 \%)$ of retear in the arthroscopic repair group and 3 cases $(6.7 \%)$ of tear aggravation in the conservative treatment group. Retear occurred in 4 patients with the high-grade partialthickness tears and in 8 with the small-to-medium-sized full-thickness tears $(\mathrm{p}=1.00)$. Tear progression occurred in 2 patients with high-grade partial-thickness tears and 1 with a small-to-medium-sized full-thickness tear $(\mathrm{p}=0.57)$. Retear and tear progression were not associated significantly with sex, age $>60$, tear on dominant side, trauma history, symptom onset or tear classification.

Table 5 showed that the proportion of those with aggravation for pain and ROM was not significantly different between the conservative treatment and arthroscopic repair groups. There was only an association with increased risk for pain aggravation in males of the conservative treatment group $(\mathrm{p}=0.034)$. Other factors such as age, tear on dominant side, pain onset, trauma history, and tear classification were not significantly associated with aggravation of pain and ROM in both groups. In the subgroup analysis of the arthroscopic repair group, the aggravation of pain assessment score and ROM was not significantly different between the groups with and without postoperative pain medication, corticosteroid injection or shoulder exercise. Retear in the arthroscopic repair group and tear progression in the conservative treatment group were not associated significantly with the aggravation of pain and ROM. 
Table 1. Patient demographics at the baseline $(\mathrm{n}=357)$

\begin{tabular}{|c|c|c|c|}
\hline & $\begin{array}{c}\text { Conservative } \\
\text { treatment }^{a)} \\
(\mathbf{n}=\mathbf{1 8 3})\end{array}$ & $\begin{array}{c}\text { Arthroscopic } \\
\left.\text { repair }^{a}\right) \\
(n=174)\end{array}$ & p-value \\
\hline Age (<60 yr) & $61(33.3)$ & $61(35.1)$ & 0.73 \\
\hline Sex (male) & $62(33.9)$ & $60(34.5)$ & 0.90 \\
\hline Tear on dominant side & $43(67.2)$ & $119(74.8)$ & 0.25 \\
\hline \multicolumn{4}{|l|}{ Pain onset } \\
\hline Acute (vs. chronic) & $43(26.2)$ & $51(29.7)$ & 0.54 \\
\hline Trauma history & $40(31.3)$ & $40(24.7)$ & 0.22 \\
\hline \multicolumn{4}{|l|}{ Tear classification } \\
\hline Partial (vs. full, $<3 \mathrm{~cm}$ ) & $69(39.2)$ & $59(34.1)$ & 0.32 \\
\hline \multicolumn{4}{|l|}{ Fatty degeneration } \\
\hline Supraspinatus & & & 0.82 \\
\hline Grade $0-1$ & $46(43.0)$ & $60(40.5)$ & \\
\hline Grade 2 & $55(51.4)$ & $77(52.0)$ & \\
\hline Grade 3-4 & $6(5.6)$ & $11(7.4)$ & \\
\hline Infraspinatus & & & 0.58 \\
\hline Grade $0-1$ & $95(88.8)$ & $133(90.5)$ & \\
\hline Grade 2 & $12(11.2)$ & $13(8.8)$ & \\
\hline Grade $3-4$ & $0(0.0)$ & $1(0.7)$ & \\
\hline Subscapularis & & & 0.67 \\
\hline Grade $0-1$ & $95(88.8)$ & $132(91.0)$ & \\
\hline Grade 2 & $12(11.2)$ & $13(9.0)$ & \\
\hline Grade 3-4 & $0(0.0)$ & $0(0.0)$ & \\
\hline \multicolumn{4}{|l|}{ Treatment } \\
\hline Conservative treatment & & & NA \\
\hline Pain medication & $144(79.6)$ & & \\
\hline Corticosteroid injection & $78(43.1)$ & & \\
\hline Shoulder exercise & $22(12.2)$ & & \\
\hline Therapeutic modality & $4(2.2)$ & & \\
\hline Active surveillance & $7(4.0)$ & & \\
\hline Surgical repair & & & NA \\
\hline Arthroscopic repair only & & $5(2.9)$ & \\
\hline Arthroscopic repair and acromioplasty & & $105(61.0)$ & \\
\hline Arthroscopic repair and debridement & & $4(2.3)$ & \\
\hline Arthroscopic repair, acromioplasty, and debridement & & $58(33.7)$ & \\
\hline \multicolumn{4}{|l|}{ Postoperative management } \\
\hline Shoulder exercise & & $106(60.9)$ & \\
\hline Pain medication & & $104(59.8)$ & \\
\hline Corticosteroid injection & & $60(34.5)$ & \\
\hline
\end{tabular}

Values are presented as number (\%).

NA, not available.

${ }^{\text {a) }}$ The sum of proportion of the patients for each category might not be $100 \%$ due to missing data. 
(A)

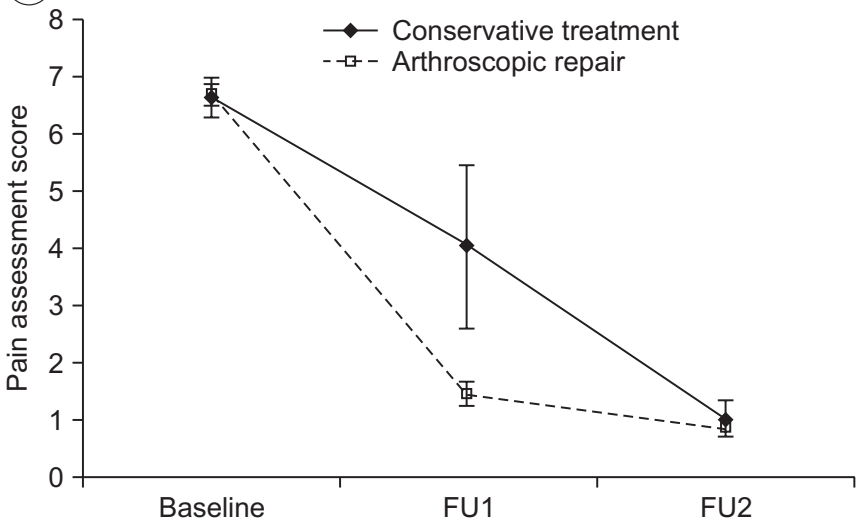

(C)

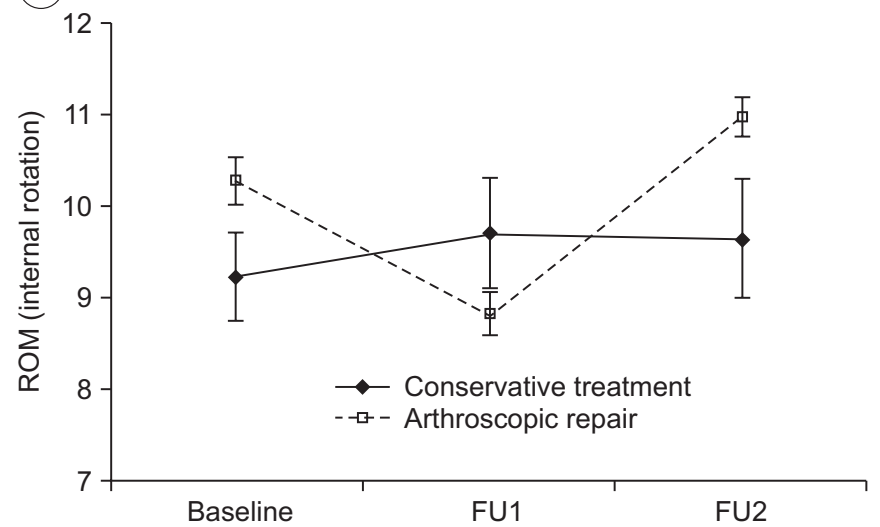

(B)

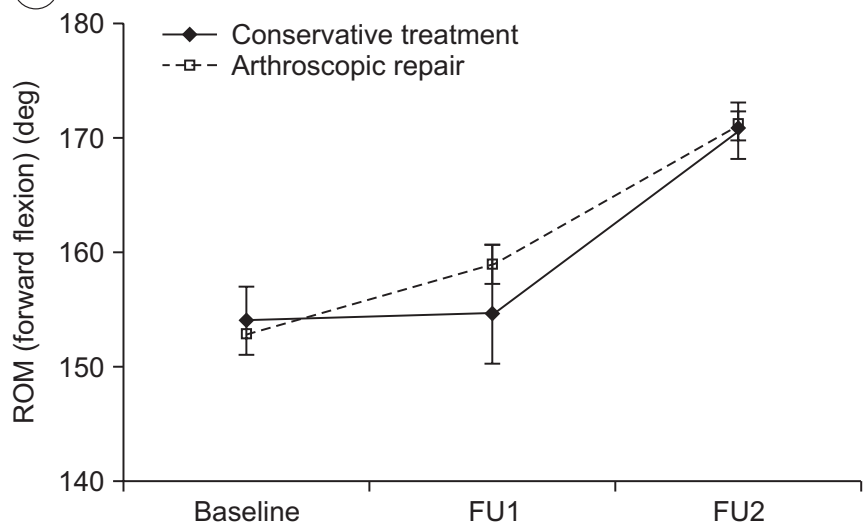

Fig. 3. (A) Pain assessment score, (B) forward flexion ROM, (C) internal rotation ROM in arthroscopic repair and conservative treatment groups at the baseline, 2-6 months (FU1) and 1 year (FU2). In the scale of internal rotation, the numerical values represent each vertebral level (i.e., 7-12 represent T12-T7 vertebral level, respectively). These values were adjusted for sex, age, tear on dominant side, symptom onset, trauma history and tear classification. ROM, range of motion; FU, follow-up.

Table 2. Clinical outcomes following conservative treatment and arthroscopic repair

\begin{tabular}{|c|c|c|c|c|c|}
\hline Clinical outcomes $^{\text {a) }}$ & $\begin{array}{c}\text { Conservative } \\
\text { treatment }\end{array}$ & $\begin{array}{c}\text { Arthroscopic } \\
\text { repair }\end{array}$ & $\begin{array}{c}\text { Between-group } \\
\text { difference }\end{array}$ & $\begin{array}{l}\text { Main } \\
\text { effect }\end{array}$ & $\begin{array}{c}\text { Treatment } x \\
\text { time interaction }\end{array}$ \\
\hline \multicolumn{6}{|l|}{ Pain } \\
\hline Baseline & $6.6 \pm 0.3$ & $6.7 \pm 0.2$ & $0.5(-0.7$ to 0.9$)$ & & \\
\hline $2-6$ months & $4.0 \pm 1.4$ & $1.4 \pm 0.2$ & $-2.6(-5.5$ to 0.3$)$ & & \\
\hline 1 year & $1.0 \pm 0.3$ & $0.9 \pm 0.2$ & $-0.2(-0.8$ to 0.5$)$ & & \\
\hline From baseline to 1 year & & & & 0.80 & 0.86 \\
\hline \multicolumn{6}{|l|}{ ROM (forward flexion) } \\
\hline Baseline & $154.1 \pm 2.9$ & $152.8 \pm 1.7$ & $-1.3(-8.0$ to 5.3$)$ & & \\
\hline $2-6$ months & $154.7 \pm 4.4$ & $159.0 \pm 1.7$ & $4.3(-5.0$ to 13.6$)$ & & \\
\hline 1 year & $170.7 \pm 2.5$ & $171.3 \pm 1.3$ & $0.5(-5.0$ to 6.1$)$ & & \\
\hline From baseline to 1 year & & & & 0.88 & 0.75 \\
\hline \multicolumn{6}{|l|}{ ROM (internal rotation) } \\
\hline Baseline & $9.2 \pm 0.5$ & $10.0 \pm 0.3$ & $1.0(0.0$ to 2.1$)$ & & \\
\hline $2-6$ months & $9.7 \pm 0.6$ & $8.8 \pm 0.2$ & $-0.9(-2.2$ to 0.4$)$ & & \\
\hline 1 year & $9.6 \pm 0.7$ & $11.0 \pm 0.2$ & $1.3(0.0$ to 2.7$)$ & & \\
\hline From baseline to 1 year & & & & 0.11 & 0.69 \\
\hline
\end{tabular}

Values are presented as mean \pm standard deviation.

${ }^{a)}$ Pain and range of motion (ROM) were adjusted for sex, age, tear on dominant side, symptom onset, trauma history and tear classification. 
Table 3. Clinical outcomes of patients with a high-grade partial-thickness tear following conservative treatment and arthroscopic repair

\begin{tabular}{|c|c|c|c|c|c|}
\hline Clinical outcomes $^{\text {a) }}$ & $\begin{array}{c}\text { Conservative } \\
\text { treatment }\end{array}$ & $\begin{array}{c}\text { Arthroscopic } \\
\text { repair }\end{array}$ & $\begin{array}{c}\text { Between-group } \\
\text { difference }\end{array}$ & $\begin{array}{l}\text { Main } \\
\text { effect }\end{array}$ & $\begin{array}{c}\text { Treatment } \times \\
\text { time interaction }\end{array}$ \\
\hline \multicolumn{6}{|l|}{ Pain } \\
\hline Baseline & $6.3 \pm 2.6$ & $6.9 \pm 2.1$ & $1.0(-0.1$ to 2.2$)$ & & \\
\hline $2-6$ months & $5.0^{\mathrm{b})}$ & $1.3 \pm 1.9$ & $-3.7(-7.7$ to 0.1$)$ & & \\
\hline 1 year & $1.1 \pm 1.7$ & $0.8 \pm 1.6$ & $-0.1(-1.1$ to 0.9$)$ & & \\
\hline From baseline to 1 year & & & & 0.22 & 0.26 \\
\hline \multicolumn{6}{|l|}{ ROM (forward flexion) } \\
\hline Baseline & $147.5 \pm 18.7$ & $150.3 \pm 21.7$ & $3.2(-7.3$ to 13.7$)$ & & \\
\hline 2-6 months & $152.3 \pm 14.1$ & $158.3 \pm 21.8$ & $6.0(-6.4$ to 18.4$)$ & & \\
\hline 1 year & $171.9 \pm 12.2$ & $168.8 \pm 15.6$ & $-4.4(-12.8$ to 4.1$)$ & & \\
\hline From baseline to 1 year & & & & 0.94 & 0.37 \\
\hline \multicolumn{6}{|l|}{ ROM (internal rotation) } \\
\hline Baseline & $8.6 \pm 3.8$ & $9.5 \pm 3.3$ & $0.8(-1.0$ to 2.6$)$ & & \\
\hline 2-6 months & $9.2 \pm 3.7$ & $8.7 \pm 2.6$ & $-0.5(-2.3$ to 1.3$)$ & & \\
\hline 1 year & $9.1 \pm 1.5$ & $11.0 \pm 2.0$ & $1.9(-0.1$ to 2.7$)$ & & \\
\hline From baseline to 1 year & & & & 0.14 & 0.64 \\
\hline
\end{tabular}

Values are presented as mean \pm standard deviation.

${ }^{\text {a) }}$ Pain and range of motion (ROM) were adjusted for sex, age, tear on dominant side, symptom onset, trauma history and tear classification.

${ }^{\text {b) }}$ The standard deviation was not calculated due to small sample size.

Table 4. Clinical outcomes of patients with a small-to-medium-sized full-thickness tear following conservative treatment and arthroscopic repair

\begin{tabular}{|c|c|c|c|c|c|}
\hline Clinical outcomes $^{\text {a) }}$ & $\begin{array}{c}\text { Conservative } \\
\text { treatment }\end{array}$ & $\begin{array}{l}\text { Arthroscopic } \\
\text { repair }\end{array}$ & $\begin{array}{l}\text { Between-group } \\
\text { difference }\end{array}$ & $\begin{array}{l}\text { Main } \\
\text { effect }\end{array}$ & $\begin{array}{c}\text { Treatment } \times \\
\text { time interaction }\end{array}$ \\
\hline \multicolumn{6}{|l|}{ Pain } \\
\hline Baseline & $7.1 \pm 1.7$ & $6.5 \pm 2.3$ & $-0.5(-1.6$ to 0.6$)$ & & \\
\hline $2-6$ months & $3.0^{\mathrm{b})}$ & $1.6 \pm 2.1$ & $-1.5(-5.8$ to 2.9$)$ & & \\
\hline 1 year & $1.0 \pm 1.3$ & $0.9 \pm 1.4$ & $-0.1(-1.1$ to 0.9$)$ & & \\
\hline From baseline to 1 year & & & & 0.44 & 0.64 \\
\hline \multicolumn{6}{|l|}{ ROM (forward flexion) } \\
\hline Baseline & $161.1 \pm 11.4$ & $154.1 \pm 20.3$ & $-6.7(-15.5$ to 2.0$)$ & & \\
\hline 2-6 months & $160.8 \pm 12.0$ & $159.6 \pm 20.3$ & $-1.2(-18.0$ to 15.5$)$ & & \\
\hline 1 year & $166.0 \pm 13.4$ & $173.5 \pm 9.7$ & $7.4(-1.9$ to 16.8$)$ & & \\
\hline From baseline to 1 year & & & & 0.95 & 0.10 \\
\hline \multicolumn{6}{|l|}{ ROM (internal rotation) } \\
\hline Baseline & $9.8 \pm 3.1$ & $10.5 \pm 3.0$ & $0.9(-0.4$ to 2.2$)$ & & \\
\hline $2-6$ months & $8.9 \pm 2.6$ & $10.8 \pm 2.5$ & $-1.9(-4.1$ to 0.3$)$ & & \\
\hline 1 year & $13.0^{\mathrm{b})}$ & $11.1 \pm 1.8$ & $-2.1(-5.7$ to 1.5$)$ & & \\
\hline From baseline to 1 year & & & & 0.75 & 0.36 \\
\hline
\end{tabular}

Values are presented as mean \pm standard deviation.

${ }^{a}$ Pain and range of motion (ROM) were adjusted for sex, age, tear on dominant side, symptom onset, trauma history and tear classification.

${ }^{\text {b) }}$ The standard deviation was not calculated due to small sample size. 
Table 5. The proportion of aggravation for pain and range of motion in conservative treatment and arthroscopic repair group (n=357)

\begin{tabular}{cccc}
\hline Aggravation of clinical outcomes $^{\text {a) }}$ & Conservative treatment & Arthroscopic repair & p-value $^{\text {b) }}$ \\
\hline Pain & $3(4.2)$ & $4(3.3)$ & 0.40 \\
ROM (forward flexion) & $11(21.6)$ & $27(24.3)$ & 0.48 \\
\hline ROM (internal rotation) & $15(62.5)$ & $49(54.4)$ & 0.26 \\
\hline
\end{tabular}

Values are presented as number (\%).

${ }^{\text {a) }}$ The sum of proportion of the patients for each category might not be $100 \%$ due to missing data.

${ }^{\text {b) }}$ Pain and range of motion (ROM) were adjusted for sex, age, tear on dominant side, symptom onset, trauma history and tear classification.

\section{DISCUSSION}

These results reveal that the clinical outcomes of conservative treatment for a rotator cuff tear are not inferior to that of arthroscopic repair of rotator cuff tears. Pain and ROM of the shoulder were not significantly different between the conservative treatment and arthroscopic repair groups at 1-year follow-up, while the clinical outcomes were improved significantly after each treatment. These clinical outcomes were not significantly different in both high-grade partial- and small-to-medium-sized full-thickness tears. Considerable cases of retear or tear progression as well as symptom aggravation after conservative or surgical treatments were identified in both groups after 1 year, but there were no significant difference between the two groups.

Conservative treatment that includes shoulder exercises, pain medication or steroid injection, has been reported to be effective for rotator cuff tears [17-19]. However, until recently, research on the effectiveness of conservative treatment compared to that of arthroscopic repair has not been published sufficiently. Two studies were carried out in 2014 to identify the superiority between shoulder exercise and arthroscopic repair with shoulder exercise or open/mini-open repair $[13,14]$. In the first study, 167 patients with a supraspinatus tear comprising $<75 \%$ of tendon insertion were provided with one of the three following treatments: shoulder exercise, shoulder exercise and acromioplasty, and shoulder exercise with acromioplasty and arthroscopic repair. The result was no significant difference of constant score at 1 year between the 3 groups. In another study, 103 patients with a full-thickness tear were treated with shoulder exercises or open/mini-open repair, and the constant score and the American Shoulder and Elbow Surgeons score were significantly different at 1 year and up to 5 years, but clinically, the difference could be negligible. The noticeable finding in this study was that the shoulder exercise group excluding the secondary repair group was not significantly different when compared with the open/miniopen group. These two studies indicated that surgical repair was not superior to shoulder exercise only, which is consistent with the result of our study. They also implied that it is important to select a patient group to show better outcome with surgical repair than conservative treatment.

According to the study on the natural course of asymptomatic rotator cuff tears, a higher percentage of patients with symptom development showed tear progression as compared with patients without symptoms $[4,7]$. The size of tendon tear did not seem to decrease, and it was shown that a partial-thickness tear can progress to a fullthickness tear in the natural course of rotator cuff disease $[5,7]$. However, the severity of the tendon tear (e.g., number of torn tendons, degree of retraction and fatty degeneration) was reported not to be correlated with the shoulder symptoms [9]. Our findings also showed that it did not determine the treatment effect and failure in terms of pain and ROM. Furthermore, this study showed that the proportion of tear progression was not different from that of retear after surgical repair. Physiotherapy was revealed to be clinically equivalent to surgical repair for shoulder pain and disability $[13,14]$. In consideration of all these aspects of rotator cuff tear, our study provides evidence that surgical repair may not be the optimal treatment of choice, based mainly upon the morphologic severity in symptomatic rotator cuff tears.

Although conservative treatment has been reported to be effective for rotator cuff tears $[8,20,21]$, a study on the optimal combination has not previously been under- 
taken. It is difficult to define and standardize conservative treatment due to its diversity and the heterogeneity of treatment methods. Additionally, each treatment may have a different rationale for symptom relief and tendon healing, but the treatment mechanisms have not yet been fully elucidated. Under these circumstances, we have tried to define conservative treatment for comparison with surgical treatment. In this study, conservative treatment was regarded as an oppositional therapeutic modality compared to surgical repair in that attachment of a torn rotator cuff tendon to the humeral head is not formed. Therefore, it could include active surveillance, pain medication, shoulder exercise and steroid injection. Further studies to find the optimal combination for conservative treatment are necessary in order to maximize treatment effectiveness and to build a personalized approach relating to individual shoulder impairment and functional status.

There were several limitations in this study. First, a degree of missing data was possible because this study was performed retrospectively based on electronic medical records. Telephone interviews were conducted to collect the additive data for the patients who were lost to follow-up, but this could also have limitations. Second, the follow-up period was only 1 year, which may not be enough to assess the long-term clinical outcome of rotator cuff tears. Third, because of the retrospective nature of this study, tear evaluation including ultrasonography, shoulder MRI or MR arthrography was not controlled. In this study, rotator cuff tear was simply divided into two categories as high-grade partial-thickness or small-tomedium-sized $(\leq 3 \mathrm{~cm})$ full-thickness tears because of this limitation. Fourth, clinical outcomes such as pain assessment score as well as forward flexion and internal rotation ROM may be not be sufficient to assess the shoulder impairment and functional status.

In conclusion, these results suggest that the effectiveness of conservative treatment is not inferior to arthroscopic repair in patients aged $>50$ years with a less than medium-sized rotator cuff tear at 1-year follow-up. The severity of tendon tear does not determine the treatment effect and failure. Further long term and prospective studies are warranted to find the optimal conservative treatment combination for maximizing the treatment effectiveness and to identify the factors that may help establish a treatment guideline for conservative treatment or surgical repair for symptomatic rotator cuff tears.

\section{CONFLICT OF INTEREST}

No potential conflict of interest relevant to this article was reported.

\section{ACKNOWLEDGMENTS}

This study was funded by National Evidence-based Healthcare Collaborating Agency (No. NECA-C-14-005).

\section{REFERENCES}

1. Tempelhof S, Rupp S, Seil R. Age-related prevalence of rotator cuff tears in asymptomatic shoulders. J Shoulder Elbow Surg 1999;8:296-9.

2. Tashjian RZ. Epidemiology, natural history, and indications for treatment of rotator cuff tears. Clin Sports Med 2012;31:589-604.

3. Pedowitz RA, Yamaguchi K, Ahmad CS, Burks RT, Flatow EL, Green A, et al. Optimizing the management of rotator cuff problems. J Am Acad Orthop Surg 2011;19:368-79.

4. Safran O, Schroeder J, Bloom R, Weil Y, Milgrom C. Natural history of nonoperatively treated symptomatic rotator cuff tears in patients 60 years old or younger. Am J Sports Med 2011;39:710-4.

5. Maman E, Harris C, White L, Tomlinson G, Shashank M, Boynton E. Outcome of nonoperative treatment of symptomatic rotator cuff tears monitored by magnetic resonance imaging. J Bone Joint Surg Am 2009;91: 1898-906.

6. Kamath G, Galatz LM, Keener JD, Teefey S, Middleton W, Yamaguchi K. Tendon integrity and functional outcome after arthroscopic repair of high-grade partial-thickness supraspinatus tears. J Bone Joint Surg Am 2009;91:105562.

7. Yamaguchi K, Tetro AM, Blam O, Evanoff BA, Teefey SA, Middleton WD. Natural history of asymptomatic rotator cuff tears: a longitudinal analysis of asymptomatic tears detected sonographically. J Shoulder Elbow Surg 2001;10:199-203.

8. Kijima H, Minagawa H, Nishi T, Kikuchi K, Shimada Y. Long-term follow-up of cases of rotator cuff tear treated conservatively. J Shoulder Elbow Surg 2012;21:491-4.

9. Dunn WR, Kuhn JE, Sanders R, An Q, Baumgarten KM, Bishop JY, et al. Symptoms of pain do not correlate with 
rotator cuff tear severity: a cross-sectional study of 393 patients with a symptomatic atraumatic full-thickness rotator cuff tear. J Bone Joint Surg Am 2014;96:793-800.

10. Lindley K, Jones GL. Outcomes of arthroscopic versus open rotator cuff repair: a systematic review of the literature. Am J Orthop (Belle Mead NJ) 2010;39:592-600.

11. Yamaguchi K, Levine WN, Marra G, Galatz LM, Klepps S, Flatow EL. Transitioning to arthroscopic rotator cuff repair: the pros and cons. Instr Course Lect 2003;52: 81-92.

12. Nho SJ, Shindle MK, Sherman SL, Freedman KB, Lyman S, MacGillivray JD. Systematic review of arthroscopic rotator cuff repair and mini-open rotator cuff repair. J Bone Joint Surg Am 2007;89 Suppl 3:127-36.

13. Kukkonen J, Joukainen A, Lehtinen J, Mattila KT, Tuominen EK, Kauko T, et al. Treatment of non-traumatic rotator cuff tears: a randomised controlled trial with one-year clinical results. Bone Joint J 2014;96B:75-81.

14. Moosmayer S, Lund G, Seljom US, Haldorsen B, Svege IC, Hennig T, et al. Tendon repair compared with physiotherapy in the treatment of rotator cuff tears: a randomized controlled study in 103 cases with a five-year followup. J Bone Joint Surg Am 2014;96:1504-14.

15. Cofield RH. Subscapular muscle transposition for repair of chronic rotator cuff tears. Surg Gynecol Obstet
1982;154:667-72.

16. Goutallier D, Postel JM, Bernageau J, Lavau L, Voisin MC. Fatty muscle degeneration in cuff ruptures: pre- and postoperative evaluation by CT scan. Clin Orthop Relat Res 1994;(304):78-83.

17. Heers G, Anders S, Werther M, Lerch K, Hedtmann A, Grifka J. Efficacy of home exercises for symptomatic rotator cuff tears in correlation to the size of the defect. Sportverletz Sportschaden 2005;19:22-7.

18. Koubaa S, Ben Salah FZ, Lebib S, Miri I, Ghorbel S, Dziri C. Conservative management of full-thickness rotator cuff tears: a prospective study of 24 patients. Ann Readapt Med Phys 2006;49:62-7.

19. Bokor DJ, Hawkins RJ, Huckell GH, Angelo RL, Schickendantz MS. Results of nonoperative management of fullthickness tears of the rotator cuff. Clin Orthop Relat Res 1993;(294):103-10.

20. Itoi E, Tabata S. Conservative treatment of rotator cuff tears. Clin Orthop Relat Res 1992;(275):165-73.

21. Kuhn JE, Dunn WR, Sanders R, An Q, Baumgarten KM, Bishop JY, et al. Effectiveness of physical therapy in treating atraumatic full-thickness rotator cuff tears: a multicenter prospective cohort study. J Shoulder Elbow Surg 2013;22:1371-9. 